ARTICLE OPEN

\title{
Large magnetoresistance and superconductivity in $\alpha$-gallium single crystals
}

\author{
Bin Chen ${ }^{1}$, Xu Duan ${ }^{1}$, Hangdong Wang ${ }^{1}$, Jianhua Du ${ }^{2}$, Yuxing Zhou ${ }^{2}$, Chunqiang Xu ${ }^{1}$, Yukun Zhang ${ }^{1}$, Liyao Zhang ${ }^{1}$, Meng Wei ${ }^{3}$,
} Zhengcai $\mathrm{Xia}^{3}$, Chao $\mathrm{Cao}^{1}{ }^{1}$, Jianhui Dai ${ }^{1}$, Minghu Fang ${ }^{2,4}$ and Jinhu Yang ${ }^{1}$

Topological metals, including Dirac and Weyl semimetals, represent a wide class of quantum materials with non-trivial electronic band structures. The essential properties of Dirac or Weyl fermions, including light effective mass and high mobility, have been observed in a number of semimetal compounds, which in turn exhibit large positive magnetoresistances. Here, we report an unexpected observation of all these properties in $a$-gallium $(a-G a)$ single crystals, a pure metal that is in the liquid phase at room temperature and ambient pressure. Based on systematical transport measurements, $a$-Ga single crystal is found to exhibit large magnetoresistance, reaching about $1.66 \times 10^{6}$ per cent at $2 \mathrm{~K}$ in a magnetic field of $9 \mathrm{~T}$. At low temperatures the de Haas-van Alphen and Shubinikov de Hass quantum oscillations show ultrahigh mobility and very small cyclotron effective mass for charge carriers, together with a non-trivial Berry phase. Combined with first-principle band structure calculations, these properties demonstrate $a-\mathrm{Ga}$ as a rare topological pure metal. Furthermore, superconductivity with $T_{\mathrm{c}}$ of $\sim 0.9 \mathrm{~K}$ is confirmed by both specific heat and resistivity measurements. These findings suggest that $a-G a$ is a unique pure metal displaying both non-trivial topological and superconducting properties.

npj Quantum Materials (2018)3:40; doi:10.1038/s41535-018-0114-3

\section{INTRODUCTION}

The recent discoveries of topological semimetals (TSMs) have stimulated intense interest in condensed matter physics and material science. ${ }^{1-6}$ The typical Dirac semimetals such as $\mathrm{Na}^{3} \mathrm{Bi}^{1,7}$ and $\mathrm{Cd} 3 \mathrm{As} 2{ }^{2}$, for instance, have four-fold degenerate band crossing points in the momentum space. The existence of these Dirac points or line-like nodes is topologically protected due to the crystal and time reversal symmetries. A Dirac semimetal can transform to a Weyl semimetal when the spin degeneracy is lifted by breaking either time-reversal or spatial inversion symmetry, resulting in paired Weyl points with opposite helicities. The inversion symmetry broken Weyl states have been discovered in a series of transition-metal monopnictide. ${ }^{5,6,8-10}$ When the Dirac or Weyl points are near the Fermi level, the relativistic Dirac or Weyl fermions emerge as low energy quasi-particles, resulting in several exotic transport phenomena such as light effective mass, high carrier mobility, and large magnetoresistance..$^{1-7,11-14}$ In general, topological insulators ${ }^{15}$ or topological superconductors ${ }^{16-26}$ can be viewed as a result of gap formation in $\mathrm{TSMs}^{7}$ without or with internal $U(1)$ symmetry breaking. More generic topological materials should include topological metals, the bulk metals with topologically protected surface states or band crossing points. Such metals can be viewed as doped TSMs or doped topological insulators, or the mixtures with additional metallic bands, though a thorough classification has yet to be reached. ${ }^{27,28}$ Among all these topological materials the two dimensional graphene is known as the simplest Dirac semimetal consisting of a single layer of pure carbons. ${ }^{29,30}$
Gallium, which was first predicted by Russian chemist Dmitri Mendeleev in 1871, is one of the four pure metals (with other three being Cesium, Rubidium, and Mercury) which are in the liquid phase near room temperature at ambient pressure. Although gallium is a simple metal element, the bulk gallium crystals have a rich phase diagram. Only the orthorhombic $a-G a$ (space group $\mathrm{Cmca}$ ) phase is stable at atmospheric pressure. In this phase, each $\mathrm{Ga}$ atom has a nearest neighbor at distance $2.44 \AA$ and six others between 2.71 and $2.79 \AA^{31}{ }^{31}$ The $a-G a$ has been long considered as a compensated metal with the broadband $s^{2} p^{1}$ electronic configuration. The Shubnikov de Hass $(\mathrm{SdH})$ and de Haas van Alphen (dHvA) quantum oscillations were measured, indicating a complicated band structure with separated Fermi surface sheets. ${ }^{32-34} \mathrm{~A}$ signal of superconducting transition below $1.08 \mathrm{~K}$ was probed earlier by specific measurement. ${ }^{3,36}$ However, such transition is hard to be probed by conventional resistivity measurement due to the extremely low resistance.

In this article, we have carried out a comprehensive investigation of bulk properties for the $a$-Ga single crystals grown by using a super-cooled method. The most prominent new feature we have found is that the magnetoresistance (MR) has a significant enhancement in the low temperature regime, reaching $1.66 \times$ $10^{6} \%$ at $2 \mathrm{~K}$ under a field of $9 \mathrm{~T}$, and $3.84 \times 10^{6} \%$ at $4.2 \mathrm{~K}$ under a pulse magnetic field of $60 \mathrm{~T}$. The magnitude of MR at this temperature regime is much larger than those in known topological semimetals. Moderately away from this regime, the MR decreases to $500 \%$ at $30 \mathrm{~K}$. The measurements of the dHvA oscillations in the isothermal magnetization show very high quantum mobility (up to $\sim 1.17 \times 10^{4} \mathrm{~cm}^{2} / \mathrm{Ns}$ at $5 \mathrm{~K}$ ) and very light

\footnotetext{
${ }^{1}$ Hangzhou Key Laboratory of Quantum Matter, Department of Physics, Hangzhou Normal University, 310036 Hangzhou, China; ${ }^{2}$ Department of Physics, Zhejiang University, 310027 Hangzhou, China; ${ }^{3}$ Wuhan National High Magnetic Field Center, Huazhong University of Science and Technology, 430074 Wuhan, China and ${ }^{4}$ Collaborative Innovation Center of Advanced Microstructures, Nanjing University, 210093 Nanjing, China

Correspondence: Jianhui Dai (daijh@hznu.edu.cn) or Minghu Fang (mhfang@zju.edu.cn) or Jinhu Yang (20110026@hznu.edu.cn)
}

Received: 9 January 2018 Revised: 15 June 2018 Accepted: 26 July 2018

Published online: 27 August 2018 

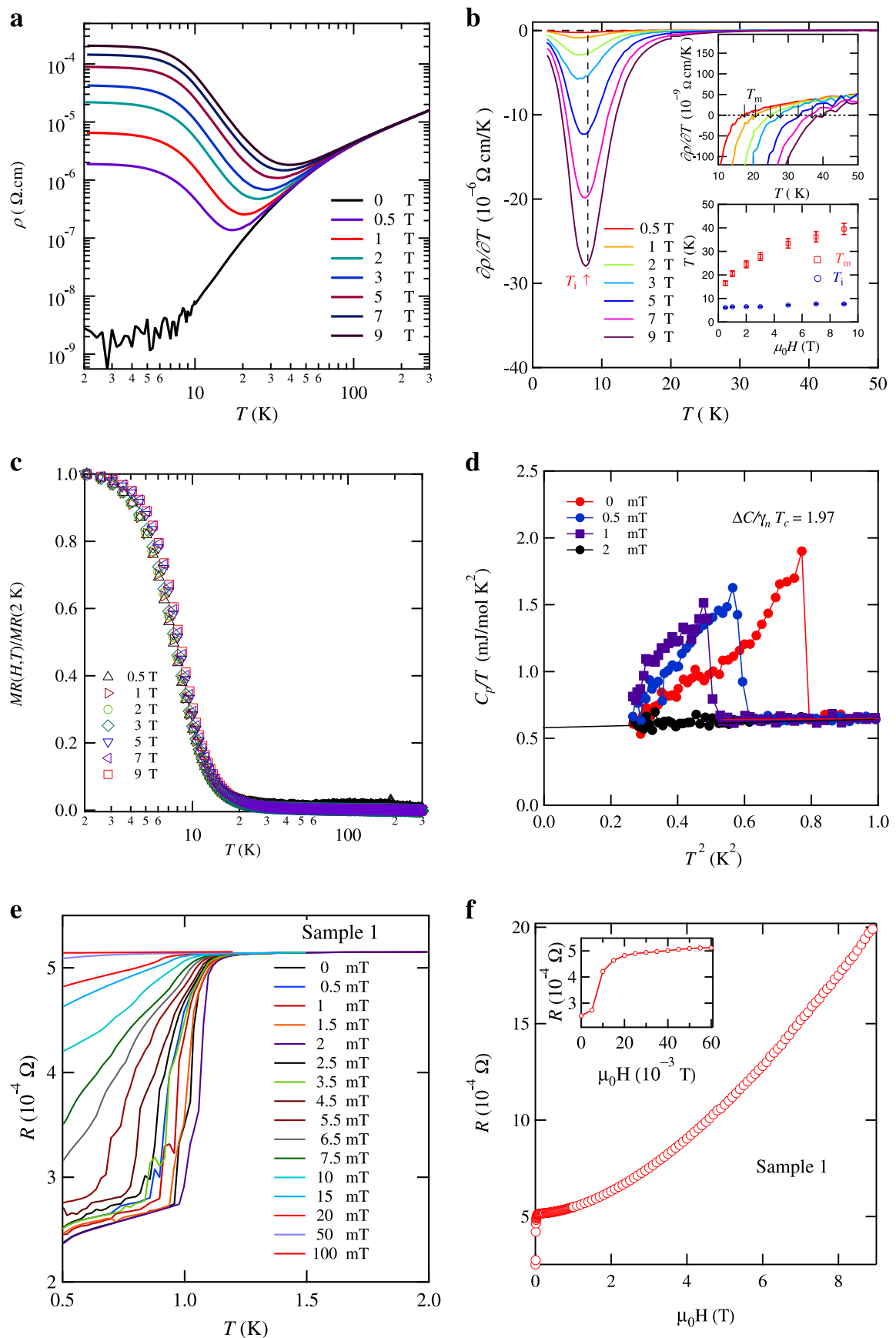

Fig. 1 Results of resistivity measurements in $\alpha$-Ga. a The log-log plot of resistivity $\rho(\mathrm{T})$ measured at various applied magnetic field $H=0,0.5$, $1,2,3,5,7$, and $9 \mathrm{~T}$ for $H / / b$-axis, the current $J$ is within the ac-plane. $\mathbf{b} \partial \rho(T) / \partial T$ plotted as a function of temperature. The minimum in the curve $\partial \rho(T) / \partial T$ marks the inflection point $T_{\mathrm{i}}$, and the sign change in the curve $\partial \rho(T) / \partial T$ marks the resistivity minimum at $T_{\mathrm{m}}$, shown in the upper inset. $T_{\mathrm{m}}$ and $T_{\mathrm{i}}$ plotted as the function of applied magnetic fields is in the low inset in which $T_{\mathrm{i}}$ stays almost unchanged whereas $T_{\mathrm{m}}$ clearly evolves with field. c The normalized magnetoresistance as a function of temperature at various magnetic fields. d Specific heat capacity divided by temperature as a function of $T^{2}$. The lambda-like peaks indicate a superconducting phase below $0.9 \mathrm{~K}$. e Resistance as a function of temperature at various magnetic fields $(0,0.5,1.5,2,2.5,3.5,4.5,5.5,6.5,7.5,10,15,20,50$, and $100 \mathrm{mT})$. f Resistance as a function of applied field at $0.5 \mathrm{~K}$. The inset displays the resistance in lower magnetic fields

effective mass $\left(\sim 0.02-0.05 m_{0}\right.$, with $m_{0}$ being the free electron mass) for charge carriers, in addition to a nontrivial Berry phase whose topological origin is evidenced by the first principle calculations. Furthermore, superconductivity in the same $a-\mathrm{Ga}$ single crystals is confirmed not only by the specific heat capacity measurement but also by an improved resistivity measurement. All these findings show the coexistence of topological phase and superconducting properties in such a simple pure metal.

\section{RESULTS}

Several $a-G a$ single crystals were measured showing the similar transport properties. We mainly report the results of "sample 1" in the following. Figure 1a shows the magnetotransport of the longitudinal resistivity $\rho(T)$ at various applied magnetic fields. In a zero magnetic field, the resistivity of the $a$-Ga single crystal at room temperature is about $16 \mu \Omega \mathrm{cm}$, nearly one order larger than that of silver, the pure metal with the best conductivity. Lowing 
Table 1. Residual resistivity, RRR, MR, and relative effective mass of the typical semimetals extracted from the relevant references

\begin{tabular}{|c|c|c|c|c|c|}
\hline Materials & $\begin{array}{l}\text { Residual } \\
\text { resistivity ( } \mu \Omega \\
\mathrm{cm})(2 \mathrm{~K})\end{array}$ & RRR & MR & $\begin{array}{l}\text { Effect mass } \\
\left(m^{*} / m_{0}\right)\end{array}$ & References \\
\hline $\mathrm{NbAs}_{2}$ & 0.2 & 317 & $8.8 \times 10^{3}$ & 0.24 & 54,55 \\
\hline $\mathrm{TaAs}_{2}$ & 0.7 & 270 & $5.8 \times 10^{3}$ & $0.08-0.14$ & 54,55 \\
\hline $\mathrm{Cd}_{3} \mathrm{As}_{2}$ & 25 & 6 & 16 & $0.02-0.04$ & 56 \\
\hline $\mathrm{WTe}_{2}$ & 2 & 370 & $1.3 \times 10^{5}$ & $\sim 0.40$ & 57 \\
\hline $\mathrm{NbP}$ & 0.63 & 115 & $8.5 \times 10^{3}$ & 0.08 & 58 \\
\hline $\mathrm{TaP}$ & 2 & 40 & $3.3 \times 10^{3}$ & $\begin{array}{l}0.04(\gamma), 0.08 \\
(\eta), 0.24(\alpha), \\
0.26(\beta)\end{array}$ & 10 \\
\hline $\mathrm{Cu}_{\mathrm{x}} \mathrm{Bi}_{2} \mathrm{Se}_{3}$ & - & - & - & 0.20 & 59 \\
\hline $\mathrm{LaSb}$ & 0.6 & 170 & $0.8 \times 10^{3}$ & 0.22 & 38 \\
\hline $\mathrm{PdTe}_{2}$ & - & - & - & $0.14-0.30$ & 23 \\
\hline $\mathrm{PtBi}_{2}$ & 0.024 & 1667 & $4 \times 10^{4}$ & $0.06-0.07$ & 60 \\
\hline $\mathrm{Na}_{3} \mathrm{Bi}$ & $1.72-87$ & $\sim 10$ & $\sim 100$ & 0.11 & 39 \\
\hline$W_{2}$ & 0.003 & 25000 & $4 \times 10^{4}$ & $\sim 1.7$ & 61 \\
\hline$a-G a$ & $\sim 0.008(10 \mathrm{~K})$ & 2000 & $1.7 \times 10^{4}$ & $0.02-0.05$ & This work \\
\hline
\end{tabular}

a

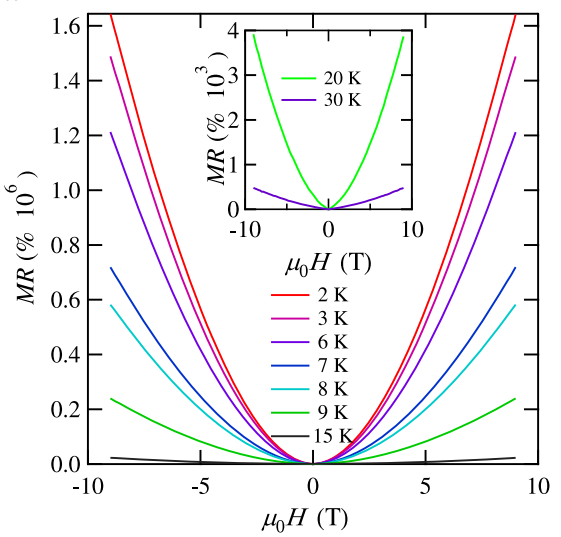

c

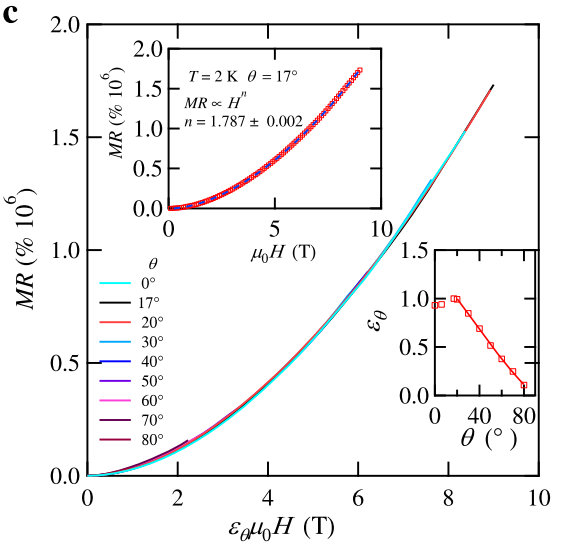

the temperature, the zero-field resistivity decreases rapidly, reaching a remarkably low residual resistivity $=8 \mathrm{n} \Omega \mathrm{cm}$ at $10 \mathrm{~K}$. The data below $10 \mathrm{~K}$ is not smooth because the magnitude of resistivity is too small to be within the limit of our PPMS measurements. The residual resistivity of the $a-G a$ single crystal is comparable with the well-known low resistivity compounds such as $\mathrm{PdCoO}_{2}(10 \mathrm{n} \Omega \mathrm{cm}, 2 \mathrm{~K}), \operatorname{IrO}_{2}(15 \mathrm{n} \Omega \mathrm{cm}, 3 \mathrm{~K})$, and $\mathrm{MoP}(6 \mathrm{n} \Omega \mathrm{cm}$, $2 \mathrm{~K}),{ }^{37}$ and is much smaller than those of topological semimetals reported so far. The detailed information is listed in Table 1. In our measurement, the residual resistivity ratio (RRR) is $\rho(300 \mathrm{~K}) / \rho(10 \mathrm{~K})$ $\approx 2000$, implying the high quality of our single crystals.

The topological characteristic of $a-\mathrm{Ga}$ is revealed by the first principle electronic band structure calculations as displayed in SIFig. 5. Without the spin-orbit coupling (SOC), the band structure of $a-\mathrm{Ga}$ shows numerous degenerate points and Dirac-like features typical for TSMs. The band crossing points at $X$, between $S$ and $R$, and between $Z$ and $\Gamma$, as well as the energy degenerate point at $Z$, are close to the Fermi level. Once the SOC is considered, all the features between the bands 12 and 13 are gapped out throughout the whole Brillouin zone. Therefore, the system could be characterized by the $Z_{2}$ topological invariant. The $Z_{2}$ classification of the system is $(1 ; 110)$, manifesting the strongly nontrivial property of the system. The topologically protected edge states are thus expected between bands 12 and 13. In addition, there is still a four-fold degenerate point for the bands 11 and 12 near the Fermi surface along the $A-Z$ direction. While the material cannot
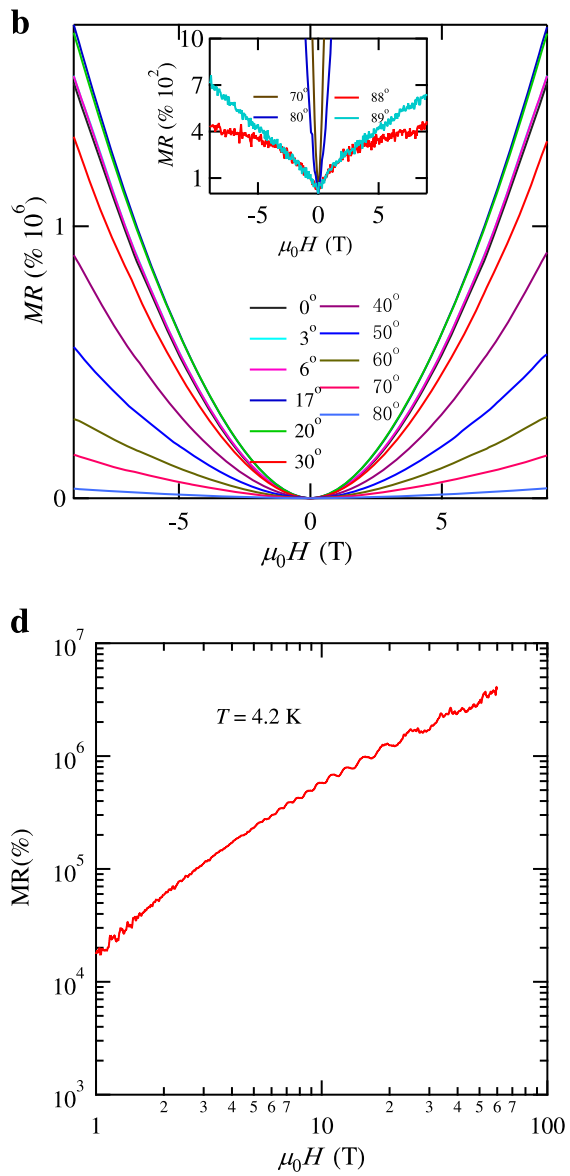

Fig. 2 Field and angular dependence of resistivity. a Temperature dependence of the magnetoresistance at various temperatures $(T=2,3,6$, $7,8,9,13,20$, and $30 \mathrm{~K}$ ) with the current parallel to $a c$-plane and the external magnetic field $H$ is parallel to $b$-axis; it is maximized at $2 \mathrm{~K}$ and $9 \mathrm{~T}$ with $\mathrm{MR}=1.66 \times 10^{6} \%$. b Angular dependence of the magnetoresistance at $2 \mathrm{~K}$ with the current within the ac-plane, and the field rotates from the $b$-axis to the ac-plane. The inset shows that the MR tends to saturate under high magnetic field when the direction of the field is close to the ac-plane. c Scaling behavior of the field dependence of the MR at various magnetic field directions. Upper inset: MR shows a power law behavior as MR $\propto H^{1.787}$ at $2 \mathrm{~K}$, lower inset: scaling factor $\varepsilon_{\theta}$ as a function of $\theta$. $\mathbf{d}$ The MR of $a$-Ga up to $60 \mathrm{~T}$ at $4.2 \mathrm{~K}$, with the current within the ac-plane and $H$ parallel to the $b$-axis 
be simply identified as a clean nodal line semimetal because of the non-integer electron filling and coexisting metallic bands, this feature may lead to the Dirac-like excitations. The detailed information is listed in SI-Table III.

Next, we turn to the results of resistivity measurements. The temperature dependence of resistivity is dramatically changed under the applied magnetic fields. A prominent field-driven resistivity up-turn appears around $20 \mathrm{~K}$ as the field increases to $0.5 \mathrm{~T}$, and, a plateau-like saturation develops below $=6 \mathrm{~K}$ in $\rho(H, T)$ as shown in Fig. 1a. In fact, this phenomenon can survive at magnetic field as low as $0.05 \mathrm{~T}$ as shown in Supplementary Information (SI-Fig. 2a). Figure $1 \mathrm{~b}$ shows the derivative $\partial \rho(T) / \partial T$ curves at various fields. In the insets of Fig. $1 \mathrm{~b}, T_{\mathrm{m}}$ is the point where $\partial \rho(T) / \partial T$ changes sign which is associated with the crossover. $T_{\mathrm{i}}$ is the inflection point of the curve below which the resistivity plateau (plotted in logarithmic scale) sets in. A subtle feature here is that $T_{\mathrm{i}}(\approx 6 \mathrm{~K})$ is nearly independent of the field whereas $T_{\mathrm{m}}$ increases with the field. Such transformative turn-on or metal-insulator-like crossover behavior has also been observed in other materials showing large $M R^{4-6}$ but at relatively high magnetic fields. Its origin is commonly attributed to the strong field and temperature dependence of high mobility of charge carriers, $^{38-40}$ or to a true metal-insulator transition or an electronic structure change driven by the magnetic field. ${ }^{41-44}$ In Fig. 1c we show a normalized MR defined as $\operatorname{MR}(H, T) / \mathrm{MR}(H, 2 \mathrm{~K})$ as a function of temperature at various magnetic fields, which collapses into one curve. This implies the fact that the plateau-like saturation observed here is field-independent, in favor of the topological origin.
When further lowing the temperature, a superconducting phase with $T_{\mathrm{c}}=0.9 \mathrm{~K}$ is observed by means of the specific heat measurement as shown in Fig. 1d. At low temperatures, $C_{\mathrm{p}} / T=$ $\gamma_{\mathrm{n}}+\beta T^{2}$, where $\gamma_{\mathrm{n}}=0.55 \mathrm{~mJ} / \mathrm{mol} \mathrm{K}^{2}$ is the Sommerfeld constant and $\beta=0.055 \mathrm{~mJ} / \mathrm{mol} \mathrm{K}^{4}$ the Debye constant. The magnitudes of $\gamma_{\mathrm{n}}$ and $\beta$ are consistent with the early reports ${ }^{35,36}$ although our $T_{\mathrm{c}}$ is a little bit smaller. The slight discrepancy in $T_{c}$ is likely due to a small residual magnetic field which cannot be removed in our PPMS equipment. It is very hard to directly detect the superconducting transition by means of resistivity measurement because the resistance of the bulk $a-\mathrm{Ga}$ is extremely low. To overcome this difficulty, we connect several bars of $a-G a$ single crystals in series so as to increase the voltage between the contacts. The temperature dependence of electrical resistance down to $0.5 \mathrm{~K}$ at ambient pressure is presented in Fig. 1e. Then, a sudden drop in resistance at $0.9 \mathrm{~K}$ is observed, in agreement with the specific heat measurement. Therefore, the drop is most reasonably caused by the superconducting transition, while the very small residual resistance down to the lowest temperature is obviously due to the electrical contact resistance between the bulk $a$-Ga bars. Upon increasing field, $T_{\mathrm{c}}$ shifts firstly to the maximum $1.1 \mathrm{~K}$ at $2.5 \mathrm{mT}$, because of the small residual magnetic field in PPMS, and then to lower temperatures. The critical magnetic field is estimated as about $5 \mathrm{mT}$ at $0.5 \mathrm{~K}$ as shown in Supplementary Information (SI-Fig. 2c).

Figure 2 shows the field dependence of the MR at various temperatures. When the current $J$ is in the ac-plane and the magnetic field is out-of-plane $(H / / b)$, the MR is positive, reaching about $1,656,500 \%$ at $2 \mathrm{~K}$ at $9 \mathrm{~T}$. This MR is large compared with the
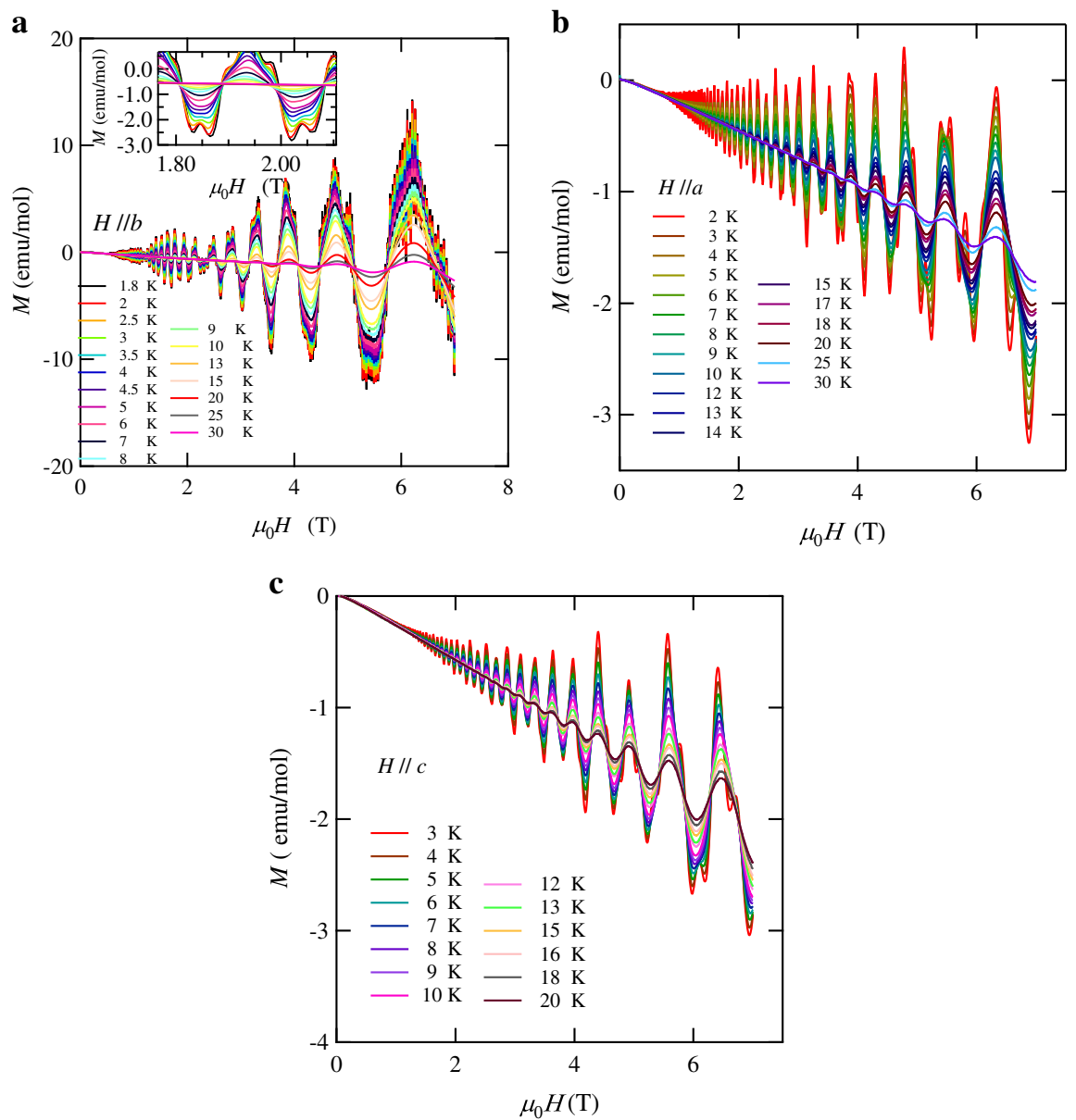

Fig. 3 Isothermal magnetization for $\mathrm{H} / / \mathrm{b}, \mathrm{H} / / \mathrm{a}$, and $\mathrm{H} / / \mathrm{c}$ in $\alpha-\mathrm{Ga}$ at various temperatures. $\mathbf{a}, \mathbf{b}, \mathbf{c}$ The oscillatory components of magnetization for $H / / b, a$ and $c$, respectively. The inset in a shows the effect of Zeeman split 

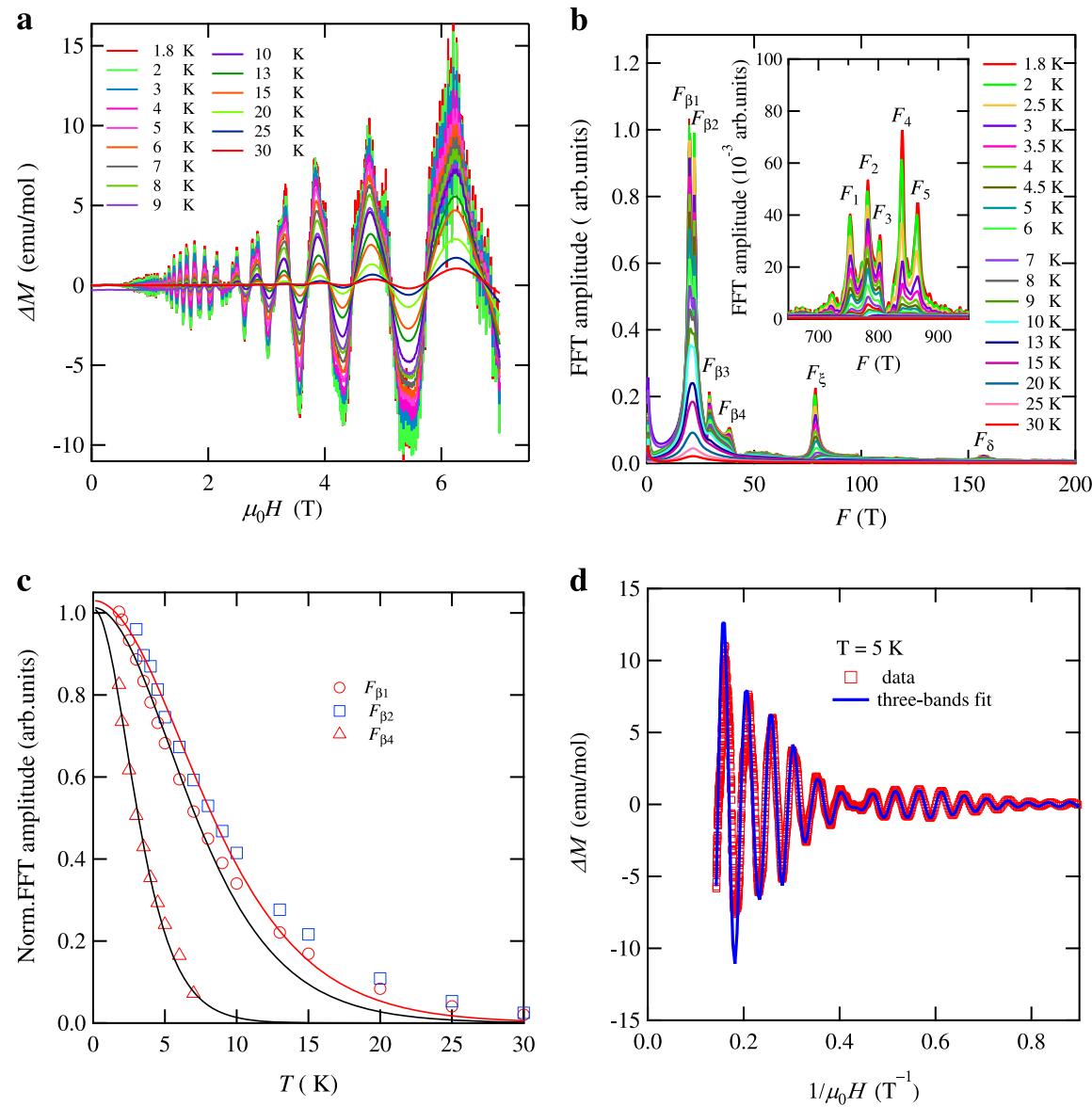

Fig. 4 Results of isothermal magnetization without background for $H / / b$. a The oscillatory components for $H / / b$. b FFT spectra of $\Delta M$ oscillations. c The normalized FFT amplitude vs. temperature; the solid lines represent the LK fits for effective mass. $\mathbf{d}$ The LK fits results at temperatures of $5 \mathrm{~K}$. The blue solid lines represent the multiband LK fits

ones in known TSMs. For instances, it is about ten times larger than that measured at the same field in $\mathrm{WTe}_{2}{ }^{3}$ and about two times of the one in $\mathrm{NbP}^{45}$ When temperature increases, the MR decreases quickly, to $500 \%$ at $30 \mathrm{~K}$ as shown in the inset of Fig. 2 a. The $M R$ follows the typical power-law $M R \propto H^{n}$ behavior, with $n$ changing from $1.787(T=2 \mathrm{~K})$ to $1.5(T=30 \mathrm{~K})$. The power law of $M R$ (with $n=2$ ) behavior may indicate a compensation effect. ${ }^{46}$ We also measured the angular dependent MR by rotating the applied field from the $b$-axis to the ac-plane, with $\theta$ being the angle between the applied field $H$ and the current $J$. The $\operatorname{MR}(H, \theta)$ at $2 \mathrm{~K}$ first increases before $17^{\circ}$ and then decreases rapidly. For example, $\mathrm{MR}\left(9 \mathrm{~T}, 88^{\circ}\right) \approx 400 \%$, which is only $0.02 \%$ of $\mathrm{MR}\left(9 \mathrm{~T}, 17^{\circ}\right)$. The power law behavior is not very sensitive to $\theta$ excepts for $\theta$ close to $90^{\circ}$ or $H / / J$ where $n$ drops to $n=0.4$. Overall, as shown in Fig. 2c, the $\operatorname{MR}(H, \theta)$ curves collapse to a single curve, $\operatorname{MR}\left(H, 17^{\circ}\right)$, with a field scaling factor $\varepsilon_{\theta} \propto \cos \left(\theta+52^{\circ}\right)$, indicating open trajectories with a single average direction in the Fermi surface. ${ }^{46}$ Moreover, as shown in Fig. $2 \mathrm{~d}$, the $M R$ increases with the field without saturation. Clear $\mathrm{SdH}$ quantum oscillations are observed at high magnetic field as shown in Fig. $2 \mathrm{~d}$.

Figure 3 presents the results of isothermal magnetization at various temperatures for $H / / b, H / / a$, and $H / / c$, respectively. Very strong oscillations with the amplitudes of $15 \mathrm{emu} / \mathrm{mol}$ at $T=2 \mathrm{~K}$ and $\mu_{0} H=7 \mathrm{~T}$ are clearly visible for $H / / b$. For $H / / a$, or $H / / c$, the oscillation amplitude is about $1 \mathrm{emu} / \mathrm{mol}$, comparable to the topological Dirac nodal-line semimetal $\mathrm{ZrSiSe}{ }^{47}$ Based on the Fast Fourier transformation (FFT) analyses, we found six major low frequencies $(19.9,22.1,29.1,38.2,78.6$, and $156.6 \mathrm{~T})$, and five major high frequencies $(753,782,803,840$, and $865 \mathrm{~T})$ for $H / / b$, see in Fig. 4b. A superposition of low-frequency and high-frequency oscillations indicated by a beat pattern is shown in Fig. 4a. The detailed frequency for $H / / a$ and $H / / C$ are presented in SI-Figs. 3b and $4 \mathrm{~b}$.

In general, the oscillatory magnetization of a Dirac system can be described by the Lifshitz-Kosevich (LK) formula ${ }^{48,49}$

$$
\Delta M \propto-B^{1 / 2} R_{\mathrm{T}} R_{\mathrm{D}} R_{\mathrm{S}} \sin \left[2 \pi\left(F / B-\varphi_{\mathrm{B}}-\delta\right)\right],
$$

here, $\varphi_{\mathrm{B}}$ is the Berry's phase, $\delta$ is a phase shift, with $\delta= \pm 1 / 8$ in 3D, $R_{\mathrm{T}}=\mathrm{aTm} * \mathrm{~B} \sinh \left(\mathrm{a} T m^{*} / B\right), \quad R_{\mathrm{D}}=\exp \left(-a T_{\mathrm{D}} m^{*} / B\right), \quad R_{\mathrm{S}}=\cos$ $\left(\pi g m^{*} / m_{0}\right), m^{*}$ and $m_{0}$ are the effective cyclotron mass and free electron mass, respectively. Here, $T_{\mathrm{D}}$ is Dingle temperature, and $a$ $=\left(2 \pi^{2} k_{\mathrm{B}} m_{0}\right) /(\hbar e) \approx 14.7 \mathrm{~T} / \mathrm{K}$. The thermal and Dingle damping terms $R_{\mathrm{T}}$ and $R_{\mathrm{D}}$ are due to the Landau level (LL) broadening driven by the finite temperature effects on the Fermi-Dirac distribution and the electron scattering rate, respectively. The spin reduction factor $R_{\mathrm{S}}$ results from the Zeeman effect. We observed the peak splits in oscillations along all three directions as shown in the inset of Fig. 4a (and SI-Figs. 3a, 4a). Raising the temperature above $10 \mathrm{~K}$, the splits gradually merge into a single peak due to the smearing of the Zeeman effect.

The effective electron cyclotron mass $m^{*}$ can be estimated by fitting the temperature dependence of the oscillation amplitude to the thermal damping factor $R_{\mathrm{T}}$, as shown in Fig. 4c (and SI-Figs. $3 c$ and $4 c$ ). For all probed oscillation frequencies, the obtained effective mass is in the range of $0.016-0.05 m_{0}$. Such value is comparable to that of the Dirac semimetal $\mathrm{Cd}_{3} \mathrm{As}_{2},{ }^{50,51}$ whose effective mass is among the smallest one in known Dirac semimetals. Taking the effective mass and the oscillation frequencies derived from the FFT spectra as known parameters, 


\begin{tabular}{|c|c|c|c|c|c|c|c|}
\hline \multirow{2}{*}{$\begin{array}{l}\text { Magnetic } \\
\text { field }\end{array}$} & \multirow[t]{2}{*}{$F(T)$} & \multirow[t]{2}{*}{$T_{\mathrm{D}}(\mathrm{K})$} & \multirow[t]{2}{*}{$\mathrm{m}^{*} / \mathrm{m}_{0}$} & \multirow[t]{2}{*}{$\tau_{\mathrm{q}(\mathrm{ps})}$} & \multirow{2}{*}{$\begin{array}{l}\mu_{\mathrm{q}} \\
\left(\mathrm{cm}^{2} /\right. \\
\mathrm{Vs})\end{array}$} & \multicolumn{2}{|l|}{$\varphi_{\mathrm{B}}(\pi)$} \\
\hline & & & & & & $\delta=1 / 8$ & $\delta=1 / 8$ \\
\hline \multirow[t]{6}{*}{$H / / b$} & 19.7 & $20(5 \mathrm{~K})$ & 0.018 & 0.62 & 5746 & 0.11 & 0.61 \\
\hline & 22.3 & $11(5 \mathrm{~K})$ & 0.016 & 1.12 & 11,787 & 0.91 & 1.41 \\
\hline & 29.1 & $35(5 \mathrm{~K})$ & 0.014 & 0.352 & 4442 & 0.99 & 0.51 \\
\hline & 38.2 & - & 0.015 & - & - & - & - \\
\hline & 78.6 & - & 0.043 & - & - & - & - \\
\hline & 156.6 & - & 0.038 & - & - & - & - \\
\hline \multirow[t]{5}{*}{$H / / a$} & 23.1 & $53(9 \mathrm{~K})$ & 0.044 & 0.232 & 915 & 0.66 & 1.02 \\
\hline & 30.1 & - & - & - & - & - & - \\
\hline & 40.4 & $37(9 \mathrm{~K})$ & 0.018 & 0.33 & 3192 & 0.51 & 1 \\
\hline & 80.7 & $40(9 \mathrm{~K})$ & 0.037 & 0.31 & 1440 & 0.87 & 0.37 \\
\hline & 133.5 & $35(9 \mathrm{~K})$ & 0.050 & 0.35 & 1221 & 1 & 0.494 \\
\hline \multirow[t]{5}{*}{$H / / C$} & 22 & $16(3 \mathrm{~K})$ & 0.049 & 0.71 & 2950 & 0.112 & 0.612 \\
\hline & 41 & $30(10 \mathrm{~K})$ & 0.017 & 0.41 & 4146 & 0.542 & 1.042 \\
\hline & & $14(3 \mathrm{~K})$ & & 0.88 & 8884 & 0.346 & 0.846 \\
\hline & 82 & $35(3 \mathrm{~K})$ & 0.026 & 0.35 & 2297 & 0.632 & 1.132 \\
\hline & 127 & $22(3 \mathrm{~K})$ & 0.040 & 0.56 & 2402 & 0.478 & 0.778 \\
\hline
\end{tabular}

we can further fit the oscillation patterns by the LK formula (Eq. 1), from which the Dingle temperature and Berry phase can be derived. To avoid the influence from the Zeeman splitting, we have fitted the $\mathrm{dHvA}$ oscillations at temperatures where the Zeeman effect is minimized by the thermal broadening of the LLs. The results are shown in Fig. 4d (and SI-Figs. 3d, 4d) and several relevant parameters obtained from the LK formula are listed in Table 2. For example, the Dingle temperature $T_{\mathrm{D}}$ is estimated as $11 \mathrm{~K}$ for $F_{\beta}=22.3 \mathrm{~T}$ at $5 \mathrm{~K}$. Therefore, the quantum relaxation time $\tau_{\mathrm{q}}=\hbar / 2 \pi k_{B} T_{\mathrm{D}}=1.12 \times 10^{-12} \mathrm{~s}$, and the quantum mobility $\mu_{\mathrm{q}}=$ $e \tau_{\mathrm{q}} / \mathrm{m}^{*}$ is estimated to be $1.17 \times 10^{4} \mathrm{~cm}^{2} /(\mathrm{Vs})$. The LK fit also yields the Berry phase $\varphi_{B}=0.91 \pi$ for $\delta=-1 / 8$, or $1.41 \pi$ for $\delta=1 / 8$. The Berry phase is close to $\pi$, thus it plays a topologically non-trivial role in the transport behavior.

We note that the magnitudes of the calculated effective masses of some bands are relatively larger than that observed by the $\mathrm{SdH}$ oscillations. The sharp dependence of Fermi surface cross section, the possible misalignment in experiments, or the magnetic breakdown effect in high magnetic fields may be the cause for the above discrepancy. Nevertheless, such discrepancy is still a puzzle and we will try to clarify it in the near future.

\section{DISCUSSION}

Materials with very large MRs are important building blocks of magnetic field sensors or other devices which are perpetually at the frontier of contemporary materials science. Meanwhile, a longstanding interest in condensed matter physics is the interplay between superconductivity and topology of electronic band structures. While the genuine topological superconductivity is expected due to this interplay, the superconductivity paring mechanism in materials with non-trivial topological band structures still remains a major puzzle. The materials exhibiting both superconductivity and topological characteristics are thus desirably attractive but rare. To our best knowledge, such candidate materials discovered so far are binary or more complicated compounds exhibiting moderately large MR. For instances, the nodal-line semimetal $\mathrm{PbTaSe}_{2}$, possessing a nonzero $Z_{2}$ topological invariant and topologically protected surface states, is a noncentrosymmetric superconductor with $T_{\mathrm{c}}=3.8 \mathrm{~K}$. Its $\mathrm{MR}$ is about $40-150 \%$ in the normal state at $9 T^{25,26}$ The type-II Dirac semimetal $\mathrm{PdTe}_{2}$ is also a superconductor with $T_{\mathrm{c}}=2 \mathrm{~K}$, the MR is $\sim 900 \%$ at $0.36 \mathrm{~K}$ under the magnetic field of $30 \mathrm{~T}$ with the effect mass of $0.13 m_{0}{ }^{52}$ A large unsaturated MR is also discovered in $\mathrm{WTe}_{2}{ }^{3}$ at ambient pressure, but the MR is quickly suppressed under high pressure, and then a superconducting state appears at the critical pressure of $10.5 \mathrm{GPa}^{21,53}$

It is known that in the type-I Dirac semimetal the density of states near the Fermi level is very small so the appearance of superconductivity usually requires electron or hole doping. The situation is different in the type-II Dirac semimetal where the density of states near the Fermi level is relatively large. ${ }^{23}$ In the Weyl semimetal, as studied in a theoretical work, ${ }^{53}$ the superconductivity may be caused by the $p_{\mathrm{x}}+i p_{\mathrm{y}}$ pairing state due to the existing Fermi arc. Combining the experimental results and the theoretical calculations, the present studied material $a-G a$ has both topological trivial and non-trivial bulk states. Therefore, such topological metal offers a particular interesting platform for understanding the superconducting mechanism in topological materials.

In summary, we have performed a systematic investigation on the high quality $a-G a$ single crystals, finding a number of fascinating properties which are unexpected for simple pure metals. The resistivity $\rho(H, T)$ of the $a$-Ga shows a plateau-like saturation at relatively low temperatures after a metal-to-insulatorlike crossover. The MR is large at low temperatures obeying the power-law behavior. The very high conductivity and large MR, together with the fitted cyclotron effect mass and the carrier mobility, manifest the $a-G a$ as a clean system with excellent transport properties among known TSMs. The electronic origin behind these properties is linked to the non-trivial topological band structure of this material, as evidenced by the observed Berry phase and the first principle calculation, suggesting the coexisting doped topological $Z_{2}$ bands and a nodal line near the Fermi level. We have also demonstrated the phenomenon of bulk superconductivity in this material by improving the resistivity measurement. Therefore, the $a-G a$ not only represents a pure metal material with interesting transport properties, but also hosts both bulk superconductivity and topological characteristics. Further investigations on the topological and surface state properties are needed in order to clarify whether this superconductivity originates from trivial or nontrivial bands. We expect that the $a-\mathrm{Ga}$ will be a new and promising platform not only for the purpose of materials application, but also for the study of the novel physics bridging the topology and superconductivity.

\section{METHODS}

XRD and single crystal growth of $a-\mathrm{Ga}$

The Ga $(99.999 \%)$ used in this work were obtained from Alfa Aesar company. The single crystal was prepared from super-cooled gallium. Large single crystals of gallium were grown from the oriented seeds. The XRD experiment was performed at room temperature with a monochromatic $\mathrm{Cu} \mathrm{K}_{\mathrm{a} 1}$ radiation using a PANalytical x-ray diffractometer (Model EMPYREAN) at Zhejiang University. We employed a conventional $\theta-2 \theta$ scan for the as grown single crystal lying on a sample holder. In order to obtain the powder XRD pattern of gallium we first paste the melted gallium to the sample holder. After cooling there are many micro-single-crystals in the surface and the XRD patterns appear no peaks or only one peak. However, we can successfully obtain the XRD pattern by carefully mixing the $\mathrm{Ga}$ powder with the Si powder below its melting temperature which ensures that the surface of Si powder enwraps gallium powder. The information about structure and XRD results are displayed in SI-Fig. 1a. The zero shift of XRD is derived as $0.0255^{\circ}$ from SI-Fig. 1b. SI-Fig. 1c displays the polycrystalline XRD pattern which is well indexed by $\mathrm{Cmca}$ space group. SI-Fig. 1d shows the single crystal XRD pattern where the crystal surface is normal to the $b$-axis. 
Theoretical calculations

The electronic band structure was calculated using plane-wave basis density functional theory (DFT) as implemented in the Vienna Ab-initio Simulation Package (VASP). Projected augmented wave (PAW) method and the Perdew, Burke, and Ernzerhof parametrization (PBE) of generalized gradient approximation were employed. An energy cut-off of $250 \mathrm{ev}$ and $12 \times 12 \times 12$ 「-centered K-mesh (over primitive cell) is sufficient to converge the total energy to $1 \mathrm{meV} /$ atom. The density of states (DOS) were obtained with dense $\Gamma$-centered K-mesh of $24 \times 24 \times 24$. The DFT band structures were fitted to a tight-binding (TB) model Hamiltonian using the maximally localized Wannier function (MLWF) method with Ga$4 p$ and $4 s$ orbitals. The Fermi surface was obtained by extrapolating the TB Hamiltonian on a $100 \times 100 \times 100 \mathrm{~K}$ mesh. Since a-Ga has an inversion center, the Z2 topological indices were calculated using the parity-check method.

\section{DATA AVAILABILITY}

All relevant data are available from the authors.

\section{ACKNOWLEDGEMENTS}

This work was financially supported by the Zhejiang Provincial Natural Science Foundation of China (Grant No. LY16A040012, LY14A040007); J. Dai was supported by the National Natural Science Foundation of China under Grant No. 11474082; Z. Xia was supported by the National Natural Science Foundation of China under Grant No. 11674115; M. Fang was supported by the National Basic Research Program of China under Grant No.2016YFA0300402. 2015CB921004) and the National Natural Science Foundation of China (Grant No. 11374261).

\section{AUTHOR CONTRIBUTIONS}

J. Yang designed the investigation. B. Chen and $X$. Duan are co-first author and contributed to this work equally. Y. Zhang, L. Zhang, and Y. Zhou synthesized the single crystals. The magnetotransport measurements in 9T PPMS were carried out by J. Du and C. Xu at Hangzhou Normal University. The pulse magnetic field measurements were conducted by $M$. Wei and Z. Xia at Wuhan National High Magnetic Field Center, Huazhong University of Science and Technology. X. Duan and C. Cao carried out the calculations. B. Chen, H. Wang, and J. Yang performed magnetization measurements and X-ray structure characterization. J. Yang, C. Cao, J. Dai, and M. Fang analyzed the results and wrote the manuscript. All authors discussed the results and commented on the manuscript.

\section{ADDITIONAL INFORMATION}

Supplementary information accompanies the paper on the npj Quantum Materials website (https://doi.org/10.1038/s41535-018-0114-3).

Competing interests: The authors declare no competing interests.

Publisher's note: Springer Nature remains neutral with regard to jurisdictional claims in published maps and institutional affiliations.

\section{REFERENCES}

1. Liu, Z. K. et al. Discovery of a three-dimensional topological dirac semimetal, $\mathrm{Na}_{3} \mathrm{Bi}$. Science 343, 864-867 (2014).

2. Liu, Z. K. et al. A stable three-dimensional topological dirac semimetal $\mathrm{Cd}_{3} \mathrm{As}_{2}$. Nat. Mater. 13, 677-681 (2014).

3. Ali, M. N. et al. Large, non-saturating magnetoresistance in $\mathrm{WTe}_{2}$. Nature 514, 205-208 (2014).

4. Tafti, F. F. et al. Resistivity plateau and extreme magnetoresistance in LaSb. Nat. Phys. 12, 272-277 (2015).

5. Yang, L. X. et al. Weyl semimetal phase in the non-centrosymmetric compound TaAs. Nat. Phys. 11, 728-732 (2015).

6. Shekhar, C. et al. Extremely large magnetoresistance and ultrahigh mobility in the topological Weyl semimetal NbP. Nat. Phys. 11, 645-649 (2015).

7. Wang, Z. et al. Dirac semimetal and topological phase transitions in $A_{3} B i(A=N a, K$ Rb). Phys. Rev. B 85, 195320 (2012).

8. Weng, $\mathrm{H}$. et al. Weyl semimetal phase in noncentrosymmetric transition-metal monophosphides. Phys. Rev. X 5, 011029 (2015).

9. $\mathrm{Xu}, \mathrm{S} . \mathrm{Y}$. et al. Discovery of a Weyl fermion state with Fermi arcs in niobium arsenide. Nat. Phys. 11, 748-754 (2015).
10. $\mathrm{Du}, \mathrm{J}$. et al. large unsaturated positive and negative magnetoresistance in weyl semimetal TaP. Sci. China Phys. Mech. Astron. 59, 657406 (2016).

11. Young, S. M. et al. Dirac semimetal in three dimensional. Phys. Rev. Lett. 108, 140405 (2012).

12. Parameswaran, D. E. et al. Probing the chiral anomaly with nonlocal transport in three-dimensional topological semimetals. Phys. Rev. X 4, 031035 (2014).

13. Hosur, P. et al. Recent development in transport phenomena in Weyl semimetals. C. R. Phys. 14, 857-850 (2013).

14. Liang, T. et al. Ultrahigh mobility and giant magnetoresistance in the Dirac semimetal $\mathrm{Cd}_{3} \mathrm{As}_{2}$. Nat. Mater. 14, 280-284 (2015).

15. Hasan, M. Z. \& Kane, C. L. Colloquium: topological insulators. Rev. Mod. Phys. 82 3045-3067 (2010).

16. Qi, X. L. \& Zhang, S. C. Topological insulators and superconductors. Rev. Mod. Phys. 83, 1057-1110 (2011).

17. Fu, L. \& Kane, C. L. Superconducting proximity effect and Majorana fermions at the surface of a topological insulator. Phys. Rev. Lett. 100, 096407 (2010).

18. Wang, $\mathrm{H}$. et al. Observation of superconductivity induced by a point contact on 3D Dirac semimetal $\mathrm{Cd}_{3} \mathrm{As}_{2}$ crystal. Nat. Mater. 15, 38-42 (2016).

19. Aggarwal, L. et al. Unconventional superconductivity at mesoscopic point contacts on the 3D Dirac semimetal $\mathrm{Cd}_{3} \mathrm{As}_{2}$. Nat. Mater. 15, 32-37 (2016).

20. Pan, X. C. et al. Pressure-driven dome-shaped superconductivity and electronic structural evolution in tungsten ditelluride. Nat. Commun. 6, 7805 (2015).

21. Qi, Y. et al. Superconductivity in Weyl semimetal candidate $\mathrm{MoTe}_{2}$. Nat. Commun 7, 11038 (2016).

22. Hor, Y. S. et al. Superconductivity in $\mathrm{Cu}_{x} \mathrm{Bi}_{2} \mathrm{Se}_{3}$ and its implications for pairing in the undoped topological insulator. Phys. Rev. Lett. 104, 057001 (2010).

23. Fei, F. et al. Nontrivial Berry phase and type-II Dirac transport in the layered material PdTe 2 . Phys. Rev. B 96, 041201(R) (2017).

24. Liu, Y. et al. Identification of topological surface state in $\mathrm{PdTe}_{2}$ superconductor by angle-resolved photoemission spectroscopy. Chin. Phys. Lett. 32, 067303 (2015).

25. Chang, T.-R. et al. Topological Dirac surface states and superconducting pairing correlations in $\mathrm{PbTaSe}_{2}$. Phys. Rev. B 93, 245130 (2016).

26. Zhang, $C$. et al. Superconducting properties in single crystals of the topological nodal semimetal in $\mathrm{PbTaSe}_{2}$. Phys. Rev. B 93, 054520 (2016).

27. Wang, J. et al. Magnetoresistance and robust resistivity plateau in MoAs2. Sci. Rep. 17, 34770 (2017).

28. Chen, J. et al. Electronic structure and topological properties of centrosymmetric $\mathrm{MoAs}_{2} / \mathrm{WAs}_{2}$ from first principles. Sci. Rep. 7, 10491 (2017).

29. Zhang, Y. et al. Experimental observational of the quantum Hall effect and Berry's phase in graphene. Nature 438, 201-204 (2005)

30. Novoselov, K. et al. Two-dimensional gas of massless dirac fermions in graphene. Nature 438, 197-200 (2005).

31. Schnering, H. G. V. et al. a-Gallium: an alternative to the Boron structure. Acta Chem. Scand. 45, 870-872 (1991).

32. Holroyd, F. W. \& Daters, W. R. de Hass-van Alphen effect in gallium. Phys. Rev. $B$ 17, 4586-4592 (1978)

33. Kimball, J. C. \& Stark, T. W. Magnetoresistance study of open orbits in gallium. Phys. Rev. B 4, 1786-1794 (1971).

34. Brown, R. L. \& Friedberg, C. B. Oscillatory magnetoresistance of gallium. Phys. Rev. B 15, 3817-3824 (1977).

35. Phillips, N. E. Low-temperature heat capacity of gallium, cadmium, and copper Phys. Rev. 134, A385-A391 (1964).

36. Gregory, W. D. Superconducting transition and critical field of pure gallium single crystals. Phys. Rev. 150, 315-321 (1966).

37. Shekhar, C. et al. Extremely high conductivity observed in the unconventional triple point fermion material MoP. arXiv:1703.03736.

38. Thoutam, L. R. et al. Temperature-dependent three-dimentional anisotropy of the magnetoresistance in $\mathrm{WTe}_{2}$. Phys. Rev. Lett. 115, 046602 (2015).

39. Wang, Y. L. et al. Origin of the turn-on temperature in $\mathrm{WTe}_{2}$. Phys. Rev. B 92, 180402(R) (2015).

40. Pei, Q. L. et al. Origin of the turn-on phenomenon in $T_{\mathrm{d}}-\mathrm{MoTe}$. Phys. Rev. B 96, 075132 (2017).

41. Zhao, Y. et al. Anisotropic magnetotransport and exotic longitudinal linear magnetoresisrance in WTe $e_{2}$ crystals. Phys. Rev. B 92, 041104 (2015).

42. Du, X. et al. Metal-insulator-like behavior in semimetallic bismuth and graphite Phys. Rev. Lett. 94, 166601 (2005).

43. Wang, K. et al. Anisotropic giant magnetoresistance in $\mathrm{NbSb}_{2}$. Sci. Rep. 4, 7328 (2014).

44. Khveshcheko, D. V. Magnetic-field-induced insulating behavior in highly oriented pyrolitic graphite. Phys. Rev. Lett. 87, 206401 (2001).

45. Shekhar, C. et al. Extremely large magnetoresistance and ultrahigh mobility in the topological Weyl semimetal candidate NbP. Nat. Phys. 11, 645-649 (2015).

46. Reed, W. A. \& Marcus, J. A. Topology of the Fermi surface of Gallium. Phys. Rev. 126, 1298 (1962) 
47. Hu, J. et al. Evidence of topological nodal-line fermions in ZrSiSe and ZrSiTe. Phys. Rev. Lett. 117, 016602 (2016).

48. Shoenberg, D. Magnetic Oscillations in Metals. (Cambridge University Press, Cambridge, 1984)

49. Lifshitz, I. M. \& Kosevich, A. M. Theory of magnetic susceptibility in metals at low temperatures. Sov. Phys. JETP 2, 636-645 (1956).

50. He, L. P. et al. quantum transport evidence for the three-dimensional Dirac semimetal phase in $\mathrm{Cd}_{3} \mathrm{As}_{2}$. Phys. Rev. Lett. 113, 246402 (2014).

51. Feng, J. et al. Large linear magnetoresistance in Dirac semimetal $\mathrm{Cd}_{3} \mathrm{As}_{2}$ with Fermi surfaces close to the Dirac points. Phys. Rev. B 92, 081306(R) (2015).

52. Wang, Y. et al. De Hass-van Alphen and magnetoresistance reveal predominantly single-band transport behavior in PdTe 2 . Sci. Rep. 6, 31554 (2016).

53. Kang, D. et al. Superconductivity emerging from a suppressed large magnetoresistance state in tungsten ditelluride. Nat. Commun. 6, 7804 (2015).

54. Wang, Y.-Y. et al. Resistivity plateau and extremely large magnetoresistance in $\mathrm{NbAs}_{2}$ and $\mathrm{TaAs}_{2}$. Phys. Rev. B 94, 041103(R) (2016).

55. Yuan, Z. et al. Large magnetoresistance in compensated semimetals $\mathrm{TaAs}_{2}$ and $\mathrm{NbAs}_{2}$. Phys. Rev. B 93, 184405 (2016).

56. Cai, P. L. et al. Drastic pressure effect on the extremely large magnetoresistance in WTe $e_{2}$ : quantum oscillation study. Phys. Rev. Lett. 15, 057202 (2015).

57. Lawson, B. J. et al. Quantum oscillations in $\mathrm{Cu}_{x} \mathrm{Bi}_{2} \mathrm{Se}_{3}$ in high magnetic fields. Phys. Rev. B 90, 195141 (2014)

58. Gao, W. et al. Extremely large magnetoresistance in a topological semimetal candidate pyrite $\mathrm{PtBi}_{2}$. Phys. Rev. Lett. 118, 256601 (2017).
59. Tafti, F. F. et al. temperature- field phase diagram of extreme magnetoresistance in lanthanum monopnictides. Proc. Natl. Acad. Sci. USA 113, E3475 (2016).

60. Xiong, J. et al. Anomalous conductivity tensor in the Dirac semimetal $\mathrm{Na}_{3} \mathrm{Bi}$. EPL 114, 27002 (2016).

61. Kumar, N. et al. Extremely high magnetoresistance and conductivity in the type-II Weyl semimetals $\mathrm{WP}_{2}$ and $\mathrm{MoP}_{2}$. Nat. Commun. 8, 1642 (2017).

(i) Open Access This article is licensed under a Creative Commons Attribution 4.0 International License, which permits use, sharing, adaptation, distribution and reproduction in any medium or format, as long as you give appropriate credit to the original author(s) and the source, provide a link to the Creative Commons license, and indicate if changes were made. The images or other third party material in this article are included in the article's Creative Commons license, unless indicated otherwise in a credit line to the material. If material is not included in the article's Creative Commons license and your intended use is not permitted by statutory regulation or exceeds the permitted use, you will need to obtain permission directly from the copyright holder. To view a copy of this license, visit http://creativecommons. org/licenses/by/4.0/.

(c) The Author(s) 2018 